\title{
On the ascending subgraph decomposition problem for bipartite graphs
}

\author{
J. M. Aroca ${ }^{1,2}$, A. Lladó ${ }^{3}$ \\ S. Slamin ${ }^{4}$ \\ Jember University, Indonesia
}

Universidad Politècnica de Catalunya, Barcelona, Spain

\begin{abstract}
The Ascending Subgraph Decomposition (ASD) Conjecture asserts that every graph $G$ with $\left(\begin{array}{c}n+1 \\ 2\end{array}\right)$ edges admits an edge decomposition $G=H_{1} \oplus \cdots \oplus H_{n}$ such that $H_{i}$ has $i$ edges and is isomorphic to a subgraph of $H_{i+1}, i=1, \ldots, n-1$. We show that every bipartite graph $G$ with $\left(\begin{array}{c}n+1 \\ 2\end{array}\right)$ edges such that the degree sequence $d_{1}, \ldots, d_{k}$ of one of the stable sets satisfies $d_{i} \geq n-i+2,1 \leq i<k$, admits an ascending subgraph decomposition with star forests. We also give a necessary condition on the degree sequence which is not far from the above sufficient one.
\end{abstract}

Keywords: Ascending subgraph deocmposition, Sumset partition problem.

\footnotetext{
1 Supported by the Catalan Research Council under project 2009SGR1387 and the Spanish Research Council under project MTM2011-28800-C02-01.

2 Email: aroca@ma4.upc.edu

3 Email: allado@ma4.upc.edu

4 Email: slamins@gmail.com
} 


\section{Introduction}

A graph $G$ with $\left(\begin{array}{c}n+1 \\ 2\end{array}\right)$ edges has an Ascending Subgraph Decomposition (ASD) if it admits an edge-decomposition $G=H_{1} \oplus \cdots \oplus H_{n}$ such that $H_{i}$ has $i$ edges and is isomorphic to a subgraph of $H_{i+1}, 1 \leq i<n$. It was conjectured by Alavi, Boals, Chartrand, Erdős and Oellerman [1] that every graph of size $\left(\begin{array}{c}n+1 \\ 2\end{array}\right)$ admits an ASD. The conjecture has been proved for a number of particular cases, including forests [5], regular graphs [9], complete multipartite graphs [8] or graphs with maximum degree $\Delta \leq n / 2$ [6].

In the same paper Alavi et al. [1] conjectured that every star forest of size $\left(\begin{array}{c}n+1 \\ 2\end{array}\right)$ in which each connected component has size at least $n$ admits an ASD in which every graph in the decomposition is a star. This conjecture was proved by Ma, Zhou and Zhou [13], and the condition was later on weakened to the effect that the second smaller component of the star forest has size at least $n$ by Chen, Fu, Wang and Zhou [4]. The above two results are connected to the Sumset Partition Problem (SPP): given an integer sequence $d_{1} \geq \cdots \geq d_{k}>0$ such that $\sum_{i} d_{i}=\left(\begin{array}{c}n+1 \\ 2\end{array}\right)$, the SPP asks for a partition $\left\{X_{1}, \ldots, X_{k}\right\}$ of the integer interval $[1, n]$ such that the sum of the elements in $X_{i}$ is precisely $d_{i}$. If the answer is positive we say that the sequence $d_{1}, \ldots, d_{k}$ is $n$-realizable. The result of Chen et al. [4] states that every sequence with $\sum_{i} d_{i}=\left(\begin{array}{c}n+1 \\ 2\end{array}\right)$ and $d_{k-1} \geq n$ is $n$-realizable.

This result can also be reformulated in terms of ASD of bipartite graphs. Let $G$ be a bipartite graph with bicoloration $\{A, B\}$ and size $\left(\begin{array}{c}n+1 \\ 2\end{array}\right)$. Suppose that the degree sequence $d_{1} \geq \cdots \geq d_{k}$ of the vertices in $A$ is $n$-realizable. Then $G$ admits a star ASD. This motivates the study of ASD for bipartite graphs in terms of the degree sequence of one of the stable sets, which is the purpose of this paper.

The condition $d_{k-1} \geq n$ given by Chen et al. [4] for a sequence to be $n$-realizable can not be weakened in the sense that the result fails to be true for sequences with $d_{k-1}=d_{k}=1$ or $d_{k-1}=d_{k}=2$, say. The two examples above belong to a family of natural obstructions for a sequence to be $n^{-}$ realizable. A sequence $a_{1} \geq \cdots \geq a_{t}$ is a forbidden sequence if there is no family of pairwise disjoint subsets $Y_{1}, \ldots, Y_{t}$ of the integer interval $\left[1, a_{1}\right]$ such that $\sum_{x \in Y_{1}}=a_{1}, \ldots, \sum_{x \in Y_{t}}=a_{t}$. Clearly, a sequence $d_{1}, \ldots, d_{k}$ which contains a forbidden sequence can not be $n$-realizable. The main result in [12] states that, for $n$ large enough, a sequence $d_{1} \geq \cdots \geq d_{k}$ with $\sum_{i} d_{i}=\left(\begin{array}{c}n+1 \\ 2\end{array}\right)$ is $n$-realizable if and only if its subsequence of terms smaller than $n$ does not contain a forbidden subsequence. The full characterization of forbidden sequences seems to be a difficult problem. In this paper we give some sufficient 
conditions which ensure the existence of a star ASD of bipartite graphs in terms of the degree sequence.

Theorem 1.1 Let $G$ be a bipartite graph with $\left(\begin{array}{c}n+1 \\ 2\end{array}\right)$ edges. Let $d_{1} \geq \cdots \geq d_{k}$ be the degree sequence of the vertices in one stable set of $G$. If $k \leq(n+1) / 4$ and $d_{k} \geq 4 k$ then $G$ admits a star $A S D$.

Most of the results on the ASD conjecture use families of graphs for which the isomorphic contention is easily checked, like matchings or stars. The main contribution of the paper is to study the conjecture for bipartite graphs in terms of the dfegree sequence of one of the stable sets by using star forests as building blocks. Our main result is the following one.

Theorem 1.2 Let $G$ be a bipartite graph with $\left(\begin{array}{c}n+1 \\ 2\end{array}\right)$ edges. Let $d_{1} \geq d_{2} \geq$ $\cdots \geq d_{k}$ be the degree sequence of one of the stable sets of $G$. If $d_{i} \geq n-i+2$ then there is a star forest $A S D$ of $G$.

The proof of Theorem 1.2 is based in a reduction lemma, which uses a deep result of Häggkvist [10] on edge-colorings, and on results on the Sumset Partition Problem [12]. The sufficient condition in Theorem 1.2 is not far from necessary.

Lemma 1.3 Let $G$ be a bipartite graph with $\left(\begin{array}{c}n+1 \\ 2\end{array}\right)$ edges. Let $d_{1} \geq d_{2} \geq \cdots \geq$ $d_{k}$ be the degree sequence of the stable set $A$ of $G$. If $G$ admits a star forest $A S D$ with the centers of the stars in $A$ then $\sum_{i=1}^{t} d_{i} \geq \sum_{i=1}^{t}(n-i+1), t=1, \ldots, k$.

The paper is organized as follows. We first consider star ASD in section 2, where we recall some results derived from the Sumset Partition Problem and prove Theorem 1.1. Section 3 contains a reduction lemma for the star forest ASD of bipartite graphs and the proof of Lemma 1.3. The proof of Theorem 1.2 is contained in section 4 .

\section{Star ASD of bipartite graphs}

A sequence $d_{1} \geq \cdots \geq d_{k}$ of nonnegative integers with $d_{1}+\cdots+d_{k}=\left(\begin{array}{c}n+1 \\ 2\end{array}\right)$ is $n$-realizable if there is a partition $\left\{X_{1}, \ldots, X_{k}\right\}$ of $[n]$ such that the sum of the elements in $X_{i}$ is $d_{i}$ for each $i$.

Let $G$ be a bipartite graph with stable sets $A=\left\{a_{1}, \ldots, a_{k}\right\}$ and $B$. Let $d_{1} \geq \ldots \geq d_{k}$ be the degrees of the vertices $a_{1}, \ldots, a_{k}$. If the sequence $\left(d_{1}, \ldots, d_{k}\right)$ is $n$-realizable then $G$ clearly admits a star $A S D$. Known results on the Sumset Partition Problem [4,12] imply the following results on the ASD problem 
Proposition 2.1 Let $G(A, B)$ be a bipartite graph with $\left(\begin{array}{c}n+1 \\ 2\end{array}\right)$ edges. If $|A| \leq$ 4 and $n \geq 7$ then $G(A, B)$ admits an $A S D$.

Chen et al. [4] proved that a sequence $\left(d_{1} \geq \cdots \geq d_{k}\right)$ such that $\sum_{i} d_{i}=$ $\left(\begin{array}{c}n+1 \\ 2\end{array}\right)$ and $d_{k-1} \geq n$ is $n$-realizable. The result can be rephrased in terms of ASD of bipartite graphs as follows.

Corollary 2.2 Let $G(A, B)$ be a bipartite graph with $\left(\begin{array}{c}n+1 \\ 2\end{array}\right)$ edges. Let $d(A)=$ $\left(d_{1} \geq \cdots \geq d_{k}\right)$ be the degree sequence of the elements in $A$. If $d_{k-1} \geq n$ then $G(A, B)$ admits a star $A S D_{n}$.

Proposition 2.3 Let $G(A, B)$ be a bipartite graph with $\left(\begin{array}{c}n+1 \\ 2\end{array}\right)$ edges. If $k \leq$ $(n+1) / 4$ and $d_{k} \geq 4 k$ then $G(A, B)$ admits a star $A S D_{n}$.

\section{A reduction Lemma}

Let $G$ be a bipartite graph with stable sets $A=\left\{a_{1}, \ldots, a_{k}\right\}$ and $B$. Let $d=\left(d_{1} \geq \cdots \geq d_{k}\right)$ be the degree sequence of the vertices in $A, d_{i}=d\left(a_{i}\right), i=$ $1, \ldots, k$. We denote by $G_{0}$ the bipartite graph with stable sets $A$ and $B^{\prime}=$ $\left\{b_{1}, \ldots, b_{d_{1}}\right\}$ where $a_{i}$ is adjacent to the vertices $b_{1}, \ldots, b_{d_{i}}, i=1, \ldots, k$, and call $G_{0}$ the compression of $G$. In this section we prove that, if $G_{0}$ admits a star forest decomposition then so does $G$. This reduces the problem of giving sufficient conditions on the degree sequence of one stable set to ensure the existence of a star forest ASD to bipartite compressed graphs. For the proof of our reduction Lemma we use the following result [10] on edge list-colorings of bipartite multigraphs.

Theorem 3.1 ([10]) Let $H$ be a bipartite multigraph with stable sets $A$ and $B$. If $H$ admits a proper edge-coloring such that each vertex $a \in A$ is incident with edges colored $\{1,2, \ldots, d(a)\}$, then $H$ can be properly edge-colored for an arbitrary assignment of lists $\{L(a): a \in A\}$ such that $|L(a)|=d(a)$ for each $a \in A$.

Lemma 3.2 (Reduction Lemma) Let $G$ be a bipartite graph with bipartition $A=\left\{a_{1}, \ldots, a_{k}\right\}$ and $B$ and degree sequence $d=\left(d_{1} \geq \cdots \geq d_{k}\right)$, $d=d\left(a_{i}\right)$, of the vertices in $A$. If the compression $G_{0}$ of $G$ admits a decomposition $G_{0}=F_{1}^{\prime} \oplus \cdots \oplus F_{t}^{\prime}$, where each $F_{i}^{\prime}$ is a star forest, then $G$ has an edge decomposition $G=F_{1} \oplus \cdots \oplus F_{t}$, where $F_{i} \cong F_{i}^{\prime}$ for each $i=1, \ldots, t$.

Proof. Let $C$ be the $(k \times t)$ matrix whose entry $c_{i j}$ is the number of edges incident to $a_{i}$ in the star forest $F_{j}^{\prime}$ of the edge decomposition of $G_{0}$. 
Consider the bipartite multigraph $H$ with $A$ and $U=\left\{u_{1}, \ldots, u_{t}\right\}$ as stable sets, where $a_{i}$ is joined with $u_{j}$ with $c_{i j}$ parallel edges. Now, for each pair $(i, j)$, color the $c_{i j}$ parallel edges of $H$ with the neighbors of $a_{i}$ in the forest $F_{j}^{\prime}$ bijectively. Note that in this way we get a proper edge-coloring of $H$ : two edges incident with a vertex $a_{i}$ receive different colors since the bipartite graph $G_{0}$ has no multiple edges, and two edges incident to a vertex $u_{j}$ receive different colors since $F_{j}^{\prime}$ is a star forest.

By the definition of the bipartite graph $G_{0}$, each vertex $a_{i} \in A$ is incident in the bipartite multigraph $H$ with edges colored $1,2, \ldots, d_{i}$. Let $L\left(a_{i}\right)$ be the list of neighbours of $a_{i}$ in the original bipartite graph $G$. By Theorem ??, there is a proper edge-coloring $\chi^{\prime}$ of $H$ in which the edges incident to vertex $a_{i}$ in $A$ receive the colors from the list $L\left(a_{i}\right)$ for each $i=1, \ldots, k$. Now construct $F_{s}$ by letting the edge $a_{i} b_{j}$ be in $F_{s}$ whenever the edge $a_{i} u_{s}$ is colored $b_{j}$ in the latter edge-coloring of $H$. Thus $F_{s}$ has the same number of edges than $F_{s}^{\prime}$ and the degree of $a_{i}$ in $F_{s}$ is $c_{i s}$, the same as in $F_{s}^{\prime}$. Moreover, since the coloring is proper, $F_{s}^{\prime}$ is a star forest. This concludes the proof.

\section{Ascending Star forest decompositions}

Let $G=G(A, B)$ be a bipartite graph with $\left(\begin{array}{c}n+1 \\ 2\end{array}\right)$ edges. We denote by $d=$ $\left(d_{1} \geq \cdots \geq d_{k}\right)$ the degree sequence of the vertices in the stable set $A$ of $G$.

We focus on star forest ASD with the stars of the decomposition centered at the vertices in $A$. We say that a degree sequence $d=\left(d_{1} \geq \cdots \geq d_{k}\right)$ with $\sum_{i} d_{i}=\left(\begin{array}{c}n+1 \\ 2\end{array}\right)$ is good if every bipartite graph $G(A, B)$ with $A=\left\{a_{1}, \ldots, a_{k}\right\}$ and $d\left(a_{i}\right)=d_{i}, 1 \leq i \leq k$, admits a star forest ASD with the centers of the stars in $A$. We next give a necessary condition for a sequence to be good.

Lemma 4.1 If the sequence $\left(d_{1} \geq \cdots \geq d_{k}\right)$ is good then

$$
\sum_{i=1}^{t} d_{i} \geq \sum_{i=1}^{t}(n-i+1) \text { for each } t=1, \ldots, k .
$$

Proof. Consider the compressed bipartite graph $G=G(A, B)$ such that $a_{i} \in$ $A$ is adjacent to $\left\{b_{j}: j=1, \ldots, d_{i}\right\}, i=1, \ldots, k$. Let $G=F_{1} \oplus \cdots \oplus F_{n}$ be a star forest ASD of $G$. Since $F_{n}$ has $n$ leaves in $B$ we clearly have $|B|=$ $d_{1} \geq n$. Thus (1) is satisfied for $t=1$. Suppose that (1) is satisfied for some $t=j-1<k$. If $d_{j} \geq n-j+1$ then the inequality extends to $t=j$. Suppose that $d_{j} \leq n-j$. Since $G$ is compressed, the neighborhhod of the vertices $a_{j+1}, \ldots, a_{k}$ is contained in the neighborhood of $a_{j}$. It follows 
that the forest $F_{n}$ has at least $\left(n-d_{j}\right)$ end-vertices adjacent only to vertices from $\left\{a_{1}, \ldots, a_{j}\right\}$. Likewise, $F_{n-i}$ has at least $\left(n-i+1-d_{j}\right)$ end-vertices adjacent only to vertices from $\left\{a_{1}, \ldots, a_{t}\right\}, i=1, \ldots, t$. Hence, $\sum_{i=1}^{j} d_{i} \geq$ $j d_{j}+\left(n-d_{j}\right)+\left(n-1-d_{j}\right)+\cdots+\left(n-t+1-d_{j}\right)=n+(n-1)+\cdots+(n-j+1)$, and (1) is satisfied for $t=j$. This concludes the proof.

We next obtain a close sufficient condition for a sequence to be good. We first introduce some definitions. Given two $k$-dimensional vectors $c=$ $\left(c_{1}, \ldots, c_{k}\right)$ and $c^{\prime}=\left(c_{1}^{\prime}, \ldots, c_{k}^{\prime}\right)$, we say that $c \preceq c^{\prime}$ if there is a permutation $\sigma \in \operatorname{Sym}(k)$ such that $c_{i} \leq c_{\sigma(i)}^{\prime}$ for $i=1,2, \ldots, k$. In other words, after reordering the components of each vector in nonincreasing order, the $i$-th component of $c$ is not larger than the $i$-th component of $c^{\prime}$. This definition is motivated by the following remark.

Remark 4.2 Let $F, F^{\prime}$ be two forests of stars with centers $x_{1}, \ldots, x_{k}$ and $x_{1}^{\prime}, \ldots, x_{k}^{\prime}$ respectively. Then $F$ is isomorphic to a subgraph of $F^{\prime}$ if and only if $\left(d_{F}\left(x_{1}\right), \ldots, d_{F}\left(x_{k}\right)\right) \preceq\left(d_{F^{\prime}}\left(x_{1}^{\prime}\right), \ldots, d_{F^{\prime}}\left(x_{k}^{\prime}\right)\right)$.

Given a sequence $d=\left(d_{1} \geq \cdots \geq d_{k}\right)$ of positive integers with $\sum_{i} d_{i}=$ $\left(\begin{array}{c}n+1 \\ 2\end{array}\right)$, we say that a $(k \times n)$ matrix $C$ with nonnegative integer entries is $d$-ascending if it satisfies the following three properties:

(A1) $\sum_{j} c_{i j}=d_{i}, i=1, \ldots, k$,

(A2) $\sum_{i} c_{i j}=n-j+1, j=1, \ldots, n$,

(A3) $c^{j} \succeq c^{j+1}, j=1, \ldots, n-1$, where $c^{j}$ denotes the $j$-th column of $C$.

Next Lemma gives a sufficient condition for a degree sequence to be good assuming the existence of an appropriate ascending matrix.

Lemma 4.3 Let $d=\left(d_{1} \geq \cdots \geq d_{k}\right)$ be a sequence of positive integers with $\sum_{i} d_{i}=\left(\begin{array}{c}n+1 \\ 2\end{array}\right)$. Suppose that there is a d-ascending matrix $C$ such that $c_{i j} \geq 1$ for each pair $(i, j)$ with $i+j \leq k+1$. If $d_{i} \geq n-i+1, i=1, \ldots, k-1$, then $d$ is good.

Proof. By the reduction Lemma it suffices to show that the compressed graph $G$ with degree sequence $d$ admits a star forest decomposition. Let $H$ be the bipartite multigraph with stable sets $A=\left\{a_{1}, \ldots, a_{k}\right\}$ and $U=\left\{u_{1}, \ldots, u_{n}\right\}$ and with $c_{i j}$ parallel edges joining $a_{i} \in A$ with $u_{j} \in U$. We next show that $H$ can be properly edge-colored in such a way that the edges incident to $a_{i}$ receive colors from the set $\left\{1, \ldots, d_{i}\right\}, i=1, \ldots, k$. Now, for each $s=1, \ldots, k$ denote by $M_{s}$ the matching in $H$ formed by the $s$ edges $a_{1} u_{s}, a_{2} u_{s-1}, \ldots, a_{s} u_{1}$. Such matchings exist by the condition $c_{i j} \geq 1$ for each pair $(i, j)$ with $i+j \leq k+1$. We color the edges of the matching $M_{s}$ with $k-s+1$. In this way the vertex $a_{i}$ is incident in $M_{1} \oplus \cdots \oplus M_{k}$ with edges colored $\{1, \ldots, k-i+1\}$. 
Let $H^{\prime}$ denote the bipartite multigraph obtained from $H$ by removing the edges in $M_{1} \oplus \cdots \oplus M_{k}$. Let $d_{A}^{\prime}=\left(d_{1}^{\prime} \geq \cdots \geq d_{k}^{\prime}\right)$ be the degree sequence of $A$ in $H^{\prime}$. Since $d_{i} \geq n-i+1$, we have $d_{i}^{\prime}=d_{i}-(k-i+1) \geq n-k, i=1, \ldots, k$. On the other hand, each vertex $u_{i}$ has degree $n-i+1$ in $H$ and, for $i \leq k$, it is incident to the matchings $M_{1}, \ldots, M_{k+1-i}$. Hence, every vertex in $U$ has degree at most $n-k$ in $H^{\prime}$.

Let $\Delta^{\prime}(A)$ be the maximum degree in $H^{\prime}$ of the vertices in $A$. If $\Delta^{\prime}(A)>$ $n-k$ then there is a matching $M_{1}^{\prime}$ in $H^{\prime}$ from the vertices of maximum degree in $A$ to $U$. Color the edges of this matching with $\Delta^{\prime}(A)$. By removing this matching from $H^{\prime}$ we obtain a bipartite multigraph in which the maximum degree of vertices in $A$ is $\Delta^{\prime}(A)-1$. By iterating this process we reach a bipartite multigraph $H^{\prime \prime}$ with $\Delta^{\prime \prime}(A)=n-k$, while the maximum degree of the vertices in $U$ still satisfies $\Delta^{\prime \prime}(U) \leq n-k$. By König's theorem, the edge-chromatic number of $H^{\prime \prime}$ is $n-k$. Hence $H^{\prime}$ can be properly edgecolored in such a way that vertex $a_{i}$ is incident in $H^{\prime}$ with colors $\left\{1, \ldots, d_{i}^{\prime}=\right.$ $\left.d_{i}-(k-i+1)\right\}$. By Theorem 3, there is also a proper edge-coloring of $H^{\prime}$ in which each vertex $a_{i}$ is incident with edges colored $\left\{k-i+1, k-i+2, \ldots, d_{i}\right\}$. By combining this coloring with the one of $M_{1} \oplus \cdots \oplus M_{k}$ defined above we get a proper edge-coloring of the original bipartite multigraph $H$ in which the vertex $a_{i}$ is incident with edges colored $\left\{1, \ldots, d_{i}\right\}$ for each $i=1, \ldots, k$.

We use this coloring to obtain a star forest decomposition $G=F_{1} \oplus \cdots \oplus F_{n}$, of the compressed bipartite graph with stable sets $A$ and $B=\left\{b_{1}, \ldots, b_{d_{1}}\right\}$ by letting $F_{s}$ consist of the edges $a_{i} b_{j}$ such that $a_{i} u_{s}$ is colored $b_{j}$ in the edgecolored multigraph $H$. Thus $F_{s}$ has degree sequence $d_{A}\left(F_{s}\right)=\left(c_{s 1}, \ldots, c_{s k}\right)$. By the column sum property of the matrix $C$, the star forest $F_{s}$ has $\sum_{i} c_{i s}=$ $n-s+1$ edges and, by the ascending column property, it is isomorphic to a subgraph of $F_{s-1}$. This completes the proof.

We are now ready to prove our main result.

Proof. Consider the matrix $C^{\prime}$ whose first row vector is $(n-k+1, \ldots, n-k+$ $1, n-k, n-k-1, \ldots 3,2,1)$ and, for $j>1$ the $i$-th row of $C^{\prime}$ has $k-i+1$ ones followed by zeros. The sum of the entries of the $j$-th column of $C^{\prime}$ is $n-i+1$. Thus $C^{\prime}$ is an ascending matrix for the degree sequence $d_{1}^{\prime}=\left(\begin{array}{c}n-k \\ 2\end{array}\right)+(n-k+1) k$ and $d_{i}^{\prime}=k-i+1$ for $i=2, \ldots, k$. Hence, $d_{i}-d_{i}^{\prime} \geq n-i+2-(k-i+1)=$ $n-k+1, i=2, \ldots, k$. Now, consider the sequence $\left(d_{2}-d_{2}^{\prime}, \ldots, d_{k}-d_{k}^{\prime}, \alpha\right)$ where $\alpha$ is such that $\sum_{i=2}^{k}\left(d_{i}-d_{i}^{\prime}\right)+\alpha=\left(\begin{array}{c}n-k+1 \\ 2\end{array}\right)$. Since all but perhaps one elements of the sequence are larger than $n-k+1$, the sequence is $(n-k+1)$-realizable. Thus there is a partition $\left\{X_{2}, \ldots, X_{k}, X_{k+1}\right\}$ of the interval $[1, n-k+1]$ with $\Sigma\left(X_{i}\right)=d_{i}-d_{i}^{\prime}, i=2, \ldots, k$. By permuting the corresponding entries from 
the first column to the $i$-th we get an ascending matrix which has row sum $d_{A}$.

\section{References}

[1] Alavi, Y., A. J.Boals, G. Chartrand, P. Erdös and O. Oellerman, The ascending subgraph decomposition problem, Congressus Numeratium 58 (1987), 7-14.

[2] Ando, K., S. Gervacio and M. Kano, Disjoint integer subsets having a constant sum, Discrete Mathematics 82 (1990), 7-11.

[3] Huaitang, Chen and Ma Kejie, On the ascending subgraph decompositions of regular graphs, Applied Mathematics - A Journal of Chinese Universities 13 (2) (1998), 165-170

[4] Chen, F. L., H. L. Fu, Y. Wang and J. Zhou. Partition of a set of integers into subsets with prescribed sums. Taiwanese Journal of Mathematics, 9 (4) (2005), 629-638.

[5] Faudree, R. J., A. Gyarfas and R. H. Schelp, Graphs which have an ascending subgraph decomposition, Congressus Numeratium 59 (1987), 49-54.

[6] Fu, H.L., A note on the ascending subgraph decomposition problem, Discrete Mathematics 84 (1990), 315-318.

[7] Fu, H. L. and W. H. Hu, A special partition of the set $I_{n}$, Bulletin of ICA 6 (1992), 57-61.

[8] Fu, H. L. and W. H. Hu, A Note on ascending subgraph decompositions of complete multipartite graphs Discrete Mathematics 226 (2001), 397-402.

[9] Fu, H. L. and W. H. Hu, Ascending subgraph decomposition of regular graphs Discrete Mathematics 253 (2002), 11 - 18.

[10] Roland, Häggkvist, Restricted edge-colourings of bipartite graphs. Combin. Probab. Comput. 5 (4) (1996), 385-392.

[11] Hamburger, P. and W. Cao, Edge Disjoint Paths of Increasing Order in Complete Bipartite Graphs, Electronic Notes in Discrete Mathematics 22 (2005) 61-67.

[12] Lladó, Anna and Jordi Moragas, On the modular sumset partition problem, European Journal of Combinatorics 33 (4) (2012), 427-434.

[13] Ma, K., H. Zhou and J. Zhou, On the ascending star subgraph decomposition of star forests, Combinatorica 14 (1994), 307-320. 